\begin{tabular}{|c|l|}
\hline Title & Effects of liquid loading on surface acoustic waves in solids \\
\hline Author(s) & Tamura, S.; V ines, R. E.; Wolfe, J. P. \\
\hline Citation & $\begin{array}{l}\text { Physical Review B, 54/7), 5151-5163 } \\
\text { https://doi.org/10.1103/PhysRevB.54.5151 }\end{array}$ \\
\hline Issue Date & 1996-08-15 \\
\hline Doc URL & http://hdl.handle.net/2115/5928 \\
\hline Rights & Copyright $\odot$ 1996 A merican Physical Society \\
\hline Type & article \\
\hline File Information & PRB54.7.pdf \\
\hline
\end{tabular}

Instructions for use 


\title{
Effects of liquid loading on surface acoustic waves in solids
}

\author{
S. Tamura \\ Department of Engineering Science, Hokkaido University, Sapporo 060, Japan \\ R. E. Vines and J. P. Wolfe \\ Department of Physics and Materials Research Laboratory, University of Illinois at Urbana-Champaign, 1110 West Green Street, \\ Urbana, Illinois 61801 \\ (Received 24 January 1996)
}

\begin{abstract}
We study the effect of liquid loading on the characteristics of surface acoustic waves propagating along the (100) surface of silicon. A nonviscous liquid layer of infinite thickness in contact with a solid substrate causes changes in the wave velocity and the attenuation of surface waves along the liquid-solid boundary. More importantly, we examine an additional branch of the Rayleigh surface that is induced by the liquid loading. We also find that the pseudosurface wave, which is restricted only to certain directions on the free surface, extends to all directions on the liquid-loaded (100) surface. Calculating the acoustic Poynting vectors associated with these surface-related acoustic modes, we find that the Rayleigh wave and the induced Rayleigh wave emit energy only into the liquid, whereas the pseudosurface wave emits energy both into the liquid and solid substrate. [S0163-1829(96)03131-1]
\end{abstract}

\section{INTRODUCTION}

The surface acoustic waves (SAW's) originally studied by Lord Rayleigh ${ }^{1}$ have attracted enormous interest of scientists in the fields of seismology, materials science, electrical engineering, and physics. ${ }^{2}$ The generalizations of Rayleigh's pioneering work, i.e., the Rayleigh waves supported on the semi-infinite, isotropic, elastic medium bounded by a stressfree planar surface, to the waves in more realistic systems, such as anisotropic media, piezoelectric media, ${ }^{3,4}$ and inhomogeneous media with solid-solid ${ }^{5}$ and solid-liquid ${ }^{6}$ boundaries have been studied extensively. However, even for the SAW's (or interface waves) at solid-liquid interfaces, important characteristics remain to be studied.

The purpose of the present work is to examine theoretically the effect of liquid loading on the propagation characteristics of SAW's on a real crystal surface with elastic anisotropy. This would be interesting in conjunction with recent experimental verifications ${ }^{7-10}$ of the predicted "phonon focusing, 11,12 at crystal surfaces. ${ }^{13-16}$ One of the current methods used for studying the focusing effect of SAW's or surface phonons utilizes focused ultrasonic immersion transducers. $^{8,9}$ With this method, the propagation of SAW's with the effect of liquid in contact with an anisotropic solid surface has been measured. Hence, the theoretical study of the SAW's in the presence of liquid loadings should be substantially important to interpret the experimental results on the SAW focusing obtained with ultrasonic immersion transducers.

It is known that even for the stress-free flat surface, the introduction of elastic anisotropy gives rise to branches of acoustic waves called pseudosurface waves (PSW's), which behave like Rayleigh surface waves (RSW's), although they are not true (nondecaying) surface waves, except in isolated directions. ${ }^{17}$ The loading of liquid on the solid surface is usually expected to act as a small perturbation on the properties of SAW's, because the acoustic impedance of a liquid $\left(Z_{L}\right)$ is typically one order of magnitude smaller than that of a solid $\left(Z_{S}\right)$ supporting SAW's, e.g., $Z_{L}=1.48 \times 10^{5} \mathrm{~g}$ $\mathrm{cm}^{-2} \mathrm{~s}^{-1}\left(Z_{L}=0.92 \times 10^{5} \mathrm{~g} \mathrm{~cm}^{-2} \mathrm{~s}^{-1}\right)$ for water (alcohol) and $Z_{S}=1.36 \times 10^{6} \mathrm{~g} \mathrm{~cm}^{-2} \mathrm{~s}^{-1}$ for the transverse mode in the $[100]$ direction of silicon. ${ }^{18}$

The effect of water loading on the Rayleigh waves propagating on the surface of steel was studied by Viktorov et al. ${ }^{6}$ They found a velocity change of about $0.05 \%$. In addition, attenuation of the Rayeigh wave occurs, due to the emission of longitudinal waves into liquid. This behavior is common, because the sound velocity $c_{L}$ in liquid is usually much smaller than the Rayleigh-wave velocity in a solid. In the case of water on top of steel, the wave amplitude is reduced to $e^{-1}$ of its original value when the Rayleigh wave travels about fourteen wavelengths along the water-steel boundary.

In the case of liquid loading on an anisotropic solid surface we predict more dramatic effects to occur, in addition to the expected small change in velocity and the occurrence of the attenuation of the SAW. Specifically, we find hiterto unobserbed branches of RSW's and PSW's that are induced by the liquid loading. These modes radiate acoustic energy either into liquid or solid, or into both. In general, those waves which radiate the energy only into the liquid retain their RSW designation; those which radiate into both liquid and solid are PSW's.

In the next section, we formulate the problem in the context of elasticity theory. In Sec. III, we give extensive numerical results on phase velocities, group velocities, and attenuation rates of the surface-related acoustic modes for alcohol and water loadings of the (001) face of silicon. Liquid loading causes additional Rayleigh modes (localized at the silicon surface) and an extension of the branches of the PSW's. The origins of these Rayleigh modes induced by liquid loading (termed IRW) and the extension of the PSW's are discussed in Sec. IV. In Sec. V, we calculate acoustic Poynting vectors to see the energy emissivities of the RSW and PSW branches. Our overall conclusions are given in Sec. VI. The origin of the IRW is analyzed more quantitatively based on fcc model in the Appendix. 


\section{FORMULATION}

We take a Cartesian coordinate system such that the solid (liquid) occupies $x_{3}=z>0(z<0)$ and the solid-liquid interface $(z=0)$ is parallel to the plane containing the vector $\mathbf{x}_{\|}=\left(x_{1}, x_{2}\right)=(x, y)$. Thus, we write the displacement vector associated with the acoustic wave in the liquid as

$$
\mathbf{U}=A \mathbf{e} e^{i\left(\mathbf{k}_{\|} \cdot \mathbf{x}_{\|}-\omega t\right)} e^{i q z},
$$

where $A$ is the amplitude, $\mathbf{k}_{\|}=\left(k_{1}, k_{2}\right)$ is the twodimensional wave vector common to that in the solid, and $q$ is the wave number in the $z$ direction which satisfies the dispersion relation

$$
\mathbf{k}_{\|}^{2}+q^{2}=\omega^{2} / c_{L}^{2} \equiv K_{L}^{2},
$$

where $K_{L}$ is the magnitude of the wave vector $\mathbf{K}_{L}$ in liquid. Only longitudinal waves can propagate in nonviscous liquid, hence, the polarization vector is written as

$$
\mathbf{e}=\left(\mathbf{k}_{\|}, q\right) / K_{L} .
$$

At the liquid-solid interface the stress $(\boldsymbol{\Sigma})$ induced by the liquid is nonvanishing only when it is normal to the interface. Thus, $\Sigma_{1}=\Sigma_{2}=0$ and

$$
\Sigma_{3}=i \lambda A\left(\mathbf{k}_{\|} \cdot \mathbf{e}_{\|}+q e_{3}\right) \equiv i A S_{3},
$$

where $S_{3}=\lambda\left(\mathbf{k}_{\|} \cdot \mathbf{e}_{\|}+q e_{3}\right)$ and $\lambda\left(=\rho_{L} c_{L}^{2}, \rho_{L}\right.$ is the density of liquid) is a Làme constant.

In a solid the displacement vector $\mathbf{u}$ takes the form

$$
\mathbf{u}=\sum_{\alpha=1}^{3} B^{(\alpha)} \boldsymbol{\varepsilon}^{(\alpha)} e^{i\left(\mathbf{k}_{\|} \cdot \mathbf{x}_{\|}-\omega t\right)} e^{i k_{3}^{(\alpha)} z},
$$

where $\alpha(=1,2$, and 3) discriminates three partial waves with amplitudes $B^{(\alpha)}$ 's, $\boldsymbol{\varepsilon}^{(\alpha)}$ 's are unit polarization vectors and $k_{3}^{(\alpha)}$ 's determine the spatial variation of the displacement perpendicular to the solid surface. It should be noted that $k_{3}^{(\alpha)}$ 's are determined by solving the equation of motion with $\mathbf{k}=\left(\mathbf{k}_{\|}, k_{3}\right)$

$$
\left(\rho_{S} \omega^{2} \delta_{i m}-C_{i j m n} k_{j} k_{n}\right) \varepsilon_{m}=0 \quad(i=1,2,3),
$$

for the given $\mathbf{k}_{\|}$and $\omega$, where $\rho_{S}$ is the mass density of the solid and $C_{i j m}$ is the stiffness tensor. The summation convention over repeated indices is assumed in Eq. (6).

The associated stress $\boldsymbol{\sigma}$ at the (001) surface of a cubic crystal is given by

$$
\begin{gathered}
\sigma_{j}=i C_{44} \sum_{\alpha=1}^{3} B^{(\alpha)}\left(k_{j} \varepsilon_{3}^{(\alpha)}+k_{3}^{(\alpha)} \varepsilon_{j}^{(\alpha)}\right) \\
\equiv i \sum_{\alpha=1}^{3} B^{(\alpha)} s_{j}^{(\alpha)} \quad(j=1,2) \\
\sigma_{3}=i \sum_{\alpha=1}^{3} B^{(\alpha)}\left[C_{12}\left(k_{1} \varepsilon_{1}^{(\alpha)}+k_{2} \varepsilon_{2}^{(\alpha)}\right)+C_{11} k_{3}^{(\alpha)} \varepsilon_{3}^{(\alpha)}\right] \\
\equiv i \sum_{\alpha=1}^{3} B^{(\alpha)} s_{3}^{(\alpha)}
\end{gathered}
$$

where

$$
\begin{gathered}
s_{j}^{(\alpha)}=C_{44}\left(k_{j} \varepsilon_{3}^{(\alpha)}+k_{3} \varepsilon_{j}^{(\alpha)}\right) \quad(j=1,2), \\
s_{3}^{(\alpha)}=C_{12}\left(k_{1} \varepsilon_{1}^{(\alpha)}+k_{2} \varepsilon_{2}^{(\alpha)}\right)+C_{11} k_{3}^{(\alpha)} \varepsilon_{3}^{(\alpha)}
\end{gathered}
$$

and $C_{i j}$ are the elastic constants. The continuity of the normal components of the stresses and the lattice displacements at the liquid-solid interface, and also the stress-free condition along the interface lead to

$$
\left|\begin{array}{cccc}
s_{1}^{(1)} & s_{1}^{(2)} & s_{1}^{(3)} & 0 \\
s_{2}^{(1)} & s_{2}^{(2)} & s_{2}^{(3)} & 0 \\
s_{3}^{(1)} & s_{3}^{(2)} & s_{3}^{(3)} & -S_{3} \\
\varepsilon_{3}^{(1)} & \varepsilon_{3}^{(2)} & \varepsilon_{3}^{(3)} & -e_{3}
\end{array}\right|=0
$$

or

$$
e_{3} \Gamma_{S}-S_{3} \Gamma_{L}=0
$$

where

$$
\Gamma_{S}=\left|\begin{array}{ccc}
s_{1}^{(1)} & s_{1}^{(2)} & s_{1}^{(3)} \\
s_{2}^{(1)} & s_{2}^{(2)} & s_{2}^{(3)} \\
s_{3}^{(1)} & s_{3}^{(2)} & s_{3}^{(3)}
\end{array}\right|
$$

and

$$
\Gamma_{L}=\left|\begin{array}{ccc}
s_{1}^{(1)} & s_{1}^{(2)} & s_{1}^{(3)} \\
s_{2}^{(1)} & s_{2}^{(2)} & s_{2}^{(3)} \\
\varepsilon_{3}^{(1)} & \varepsilon_{3}^{(2)} & \varepsilon_{3}^{(3)}
\end{array}\right|
$$

Note that the boundary condition valid for a stress-free planar surface is

$$
\Gamma_{S}=0 \text {. }
$$

The boundary condition (10) determines the velocity of surface waves $c=\omega /\left|\mathbf{k}_{\|}\right|$, with $k_{3}^{(\alpha)}$, s determined from Eq. (6). Actually, for determining a $k_{3}^{(\alpha)}$ from Eq. (6), we need to know $c$, so Eqs. (6) and (10) are used to determine $k_{3}^{(\alpha)}$, s and $c$ simultaneously. Here, we define the RSW as the mode with all three partial waves localized near the solid surface, i.e., $\operatorname{Im} k_{3}^{(\alpha)}>0$ for $\alpha=1,2$, and 3. If (at least) one of $k_{3}^{(\alpha)}$, s has a negative imaginary part, the amplitude of the corresponding wave grows in the bulk of solid at an increasing normal distance from surface. This wave corresponds to the PSW.

An additional complexity arises from the fact that Eq. (6) [together with Eq. (10)] has solutions for a complex $\mathbf{k}_{\|} \cdot{ }^{19}$ This is because the phase velocity of surface waves on the free surface of a solid is usually faster than the sound velocity $c_{L}$ in liquid and the effect of liquid loading on the surface-wave velocity is small, so that $c>c_{L}$ still holds. 


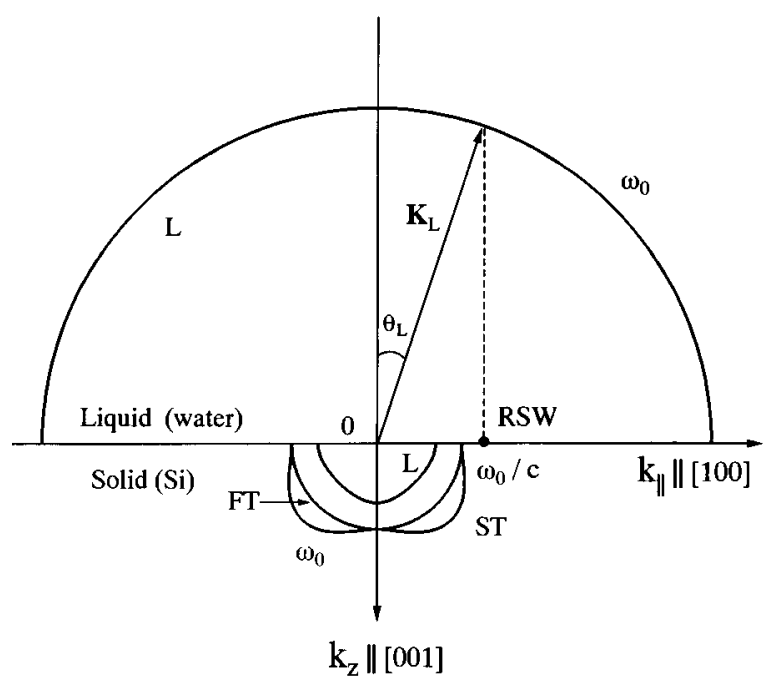

FIG. 1. The (010) section of the constant-frequency surfaces of the bulk elastic waves [longitudinal (L), fast transverse (FT), and slow transverse $(\mathrm{ST})]$ on a silicon loaded by water. The dot indicates the location of the wave number $\left(k_{\|}=\omega_{0} / c, \omega_{0}\right.$ is a given frequency) of the Rayleigh surface wave (RSW) in the [100] direction. The angle $\theta_{L}=\sin ^{-1}\left(c_{L} / c\right)$ gives the direction of the wave vector $\mathbf{K}_{L}$ along which the longitudinal wave is emitted into water from the RSW $\left[\theta_{L} \simeq 17.5^{\circ}\right.$ for water on a silicon, where $c_{L}=1.483 \times 10^{5} \mathrm{~cm} / \mathrm{s}$ and $c=4.928 \times 10^{5} \mathrm{~cm} / \mathrm{s}$ for the velocities of the longitudinal wave in water and the RSW in silicon are used].

Thus, the phase matching condition requires that a longitudinal wave is emitted from the surface wave into the liquid with an angle $\theta_{L}=\sin ^{-1}\left(c_{L} / c\right)$, as measured from the normal of the interface. This can be readily understood by plotting the slowness curves of acoustic waves in liquid and solid, as shown in Fig. 1. This figure shows the (010) sections of the constant-frequency $\left(\omega=\omega_{0}\right)$ surfaces (or slowness surfaces) of the longitudinal $(L)$ wave in liquid and the bulk acoustic waves in silicon. The velocity $c$ of an RSW propagating in the [100] direction on the free (001) silicon surface is $c=4.93 \times 10^{5} \mathrm{~cm} / \mathrm{s}$ and the corresponding slowness is $c^{-1}=2.03 \times 10^{-6} \mathrm{~s} / \mathrm{cm}$, which occurs outside the slowness curves in silicon, but inside the one in water (the dot in Fig. $1)$.

Thus, for a given frequency $\omega_{0}$, a real wave vector $\left(\mathbf{K}_{L}\right)$ exists for the wave in water, which has a parallel slowness equal to that of the RSW. However, this is not the case with the wave in solid. The surface waves on the substrate surface perturbed by a semi-infinite, nonviscous liquid are attenuated as they propagate, radiating their acoustic energy into liquid, i.e., complex $\mathbf{k}_{\|}$is required to satisfy both the elasticity equations and Snell's law. This can be also seen by explicitly writing Eq. (10) for an isotropic solid, 3,6

$$
\begin{aligned}
\left(k_{\|}^{2}\right. & \left.-K_{L}^{2}\right)^{1 / 2}\left[4 k_{\|}^{2}\left(k_{\|}^{2}-k_{t}^{2}\right)^{1 / 2}\left(k_{\|}^{2}-k_{l}^{2}\right)^{1 / 2}-\left(2 k_{\|}^{2}-k_{t}^{2}\right)^{2}\right] \\
& =\frac{\rho_{L}}{\rho_{S}} k_{t}^{4}\left(k_{\|}^{2}-k_{l}^{2}\right)^{1 / 2},
\end{aligned}
$$

where $k_{\|}=\left|\mathbf{k}_{\|}\right|, k_{l}=\omega / c_{l}$ and $k_{t}=\omega / c_{t}$, and $c_{l}$ and $c_{t}$ are the velocities of the longitudinal and transverse waves in solid defined by $c_{l}^{2}=C_{11} / \rho_{S}$ and $c_{t}^{2}=C_{44} / \rho_{S}$ under the condition $C_{11}-C_{12}=2 C_{44}$ required for an isotropic medium. For
$\rho_{L} / \rho_{S}=0$ (valid for a free surface), Eq. (14) has a real solution $k_{\|}$satisfying $K_{L}>k_{\|}>k_{t}>k_{l}$ (or $c_{L}<c<c_{t}<c_{l}$ ). This corresponds to the Rayleigh wave ( $c=\omega / k_{\|}$is its velocity). For a finite $\rho_{L} / \rho_{S}$ and $K_{L}>k_{\|}>k_{t}>k_{l}$, the right-hand side (r.h.s.) of Eq. (14) is real but the left-hand side (1.h.s.) is pure imaginary. This means that $k_{\|}$satisfying Eq. (14) should be a complex quantity.

The same thing also happens for an anisotropic solid. Thus, we put $\mathbf{k}_{\|}=\mathbf{k}_{\|}^{(R)}+i \mathbf{k}_{\|}^{(I)}$, where $\mathbf{k}_{\|}^{(I)}$ determines the attenuation (dependent on the propagation direction) of the wave along the substrate surface. The SAW velocity $c$, which is real, should be redefined by $c=\omega /\left|\mathbf{k}_{\|}^{(R)}\right|=\omega / k_{\|}^{(R)}$. We also introduce the attenuation coefficient $\tilde{\alpha}$ defined by $\tilde{\alpha}=k_{\|}^{(I)} / k_{\|}^{(R)}=\left|\mathbf{k}_{\|}^{(I)}\right| /\left|\mathbf{k}_{\|}^{(R)}\right|$. In the numerical calculations, we search for the SAW solutions by using $\mathbf{k}_{\|}=(1+i \widetilde{\alpha}) \mathbf{k}_{\|}^{(R)}$ or $\mathbf{k}_{\|}^{(I)}=\widetilde{\alpha} \mathbf{k}_{\|}^{(R)}{ }^{17}$

\section{NUMERICAL RESULTS}

Figures 2(a) and 2(b) show one of the principal results of our calculations. In these figures, we plot the phase velocities versus the propagation direction of surface waves on the (001) face of silicon. The velocities of the RSW and PSW on the free (001) face of silicon are plotted as triangles and circles, respectively. In the [110] direction $\left(45^{\circ}\right)$ the RSW degenerates into the bulk slow transverse (ST) wave at point $C$, and a true surface wave with localized character appears in the PSW branch (point $B$, see also Appendix). Also the PSW degenerates into the bulk ST mode at angles smaller than $\sim 23^{\circ}$ and no PSW solution is obtained at these angles. $^{17}$

In Fig. 2, we also plot the velocities of both the RSW and PSW on the same surface of silicon with the loading of alcohol and water layers of semi-infinite thickness. We find two kinds of qualitative changes due to liquid loading.

(i) A branch of Rayleigh surface wave [we refer to it as an induced Rayleigh wave (IRW)] appears close to the original PSW branch at angles $\geqslant 27^{\circ}$. This can be clearly seen for the case of alcohol loading. For water loading, the situation is more complicated and interesting: the IRW appears to run continuously into the RSW branch at an angle around $28^{\circ}$. For consistency with the unloaded and alcohol-loaded cases, however, we view the IRW branch as touching the RSW at a point $(D)$, leaving both branches sharply cusped. The upgoing branch of the IRW becomes nearly degenerate with the bulk ST mode.

Note that the branches that we have labeled the RSW and IRW are together part of the generalized Rayleigh-wave structure for this interface; they radiate acoustic energy only into the liquid, as we shall see in Sec. V. In the case of liquid loading, we define the branch with a phase velocity closer to that at the free surface as the RSW. So, for water loading there is a transition from the RSW branch to the IRW branch at an angle $\sim 28^{\circ}$, but no such transition occurs between the RSW and IRW for alcohol loading, justifying our terminology. The contact between branches is found to first occur for $Z_{L} / Z_{S} \simeq 0.085$.

(ii) The PSW branch, which for the free surface exists at a restricted region from an angle $\sim 23^{\circ}$ to the [110] direction, extends all the way to the [100] direction when the 

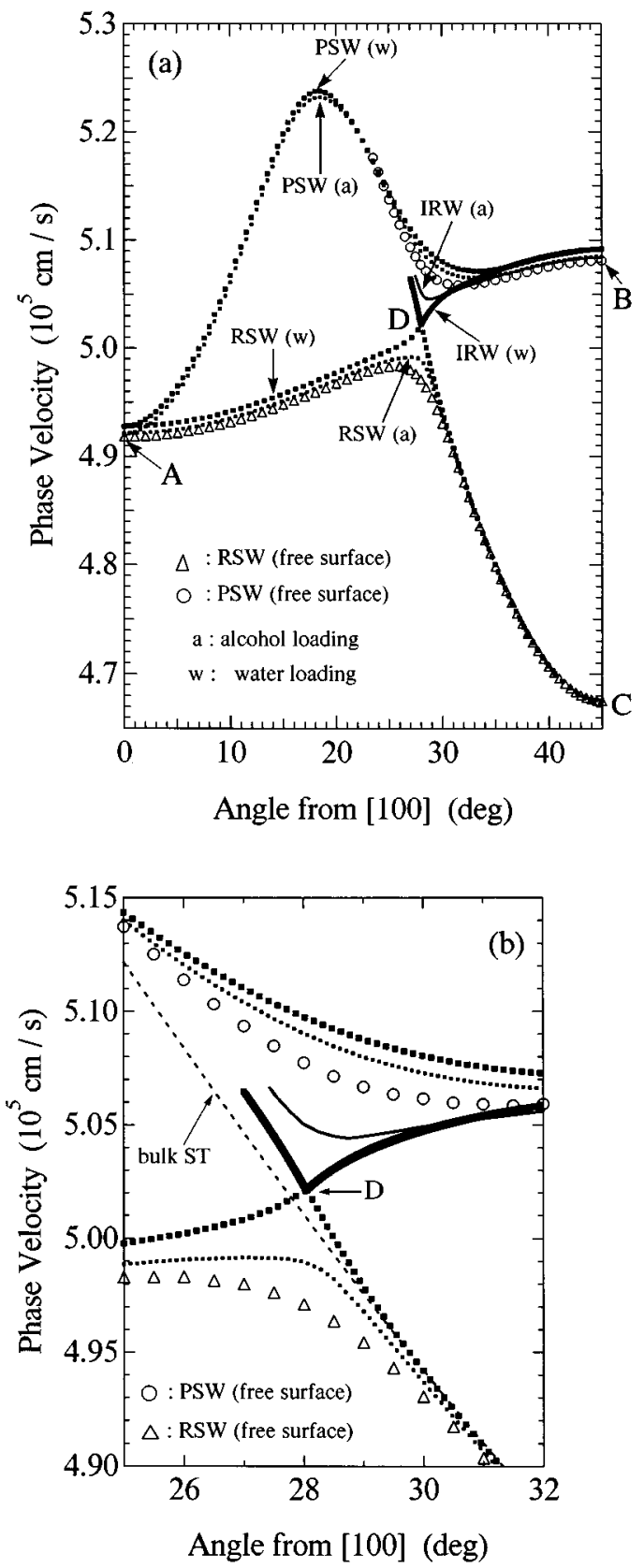

FIG. 2. (a) Phase velocities of the surface waves in the (001) plane of silicon with water and alcohol loadings. [The abscissa is the wave-vector angle measured from the [100] direction.] The velocities of the RSW and PSW are plotted by dots, while those of the IRW's are plotted by solid lines. The RSW and PSW velocities on the free silicon surface are plotted by triangles and open circles, respectively. At the points marked by " $A$ ", and " $B$,' ' the waves are polarized within the saggital plane. At point " $C$,', the RSW degenerates to a bulk shear wave (slow transverse wave) polarized horizontally. (b) The magnification of the phase velocities near the point " $D$ " of (a). The velocities of the bulk slow transverse (ST) wave are plotted by dashed line.

(001) surface is loaded by a liquid.

The group velocities corresponding to these cases are shown in Fig. 3. Figure 3(b) for the alcohol loading shows the folding of the group-velocity curve of the RSW, exhibiting cusps at $9.3^{\circ}$ and $27.2^{\circ}$ from the [100] (and their equivalent directions), which is similar to the result for the free
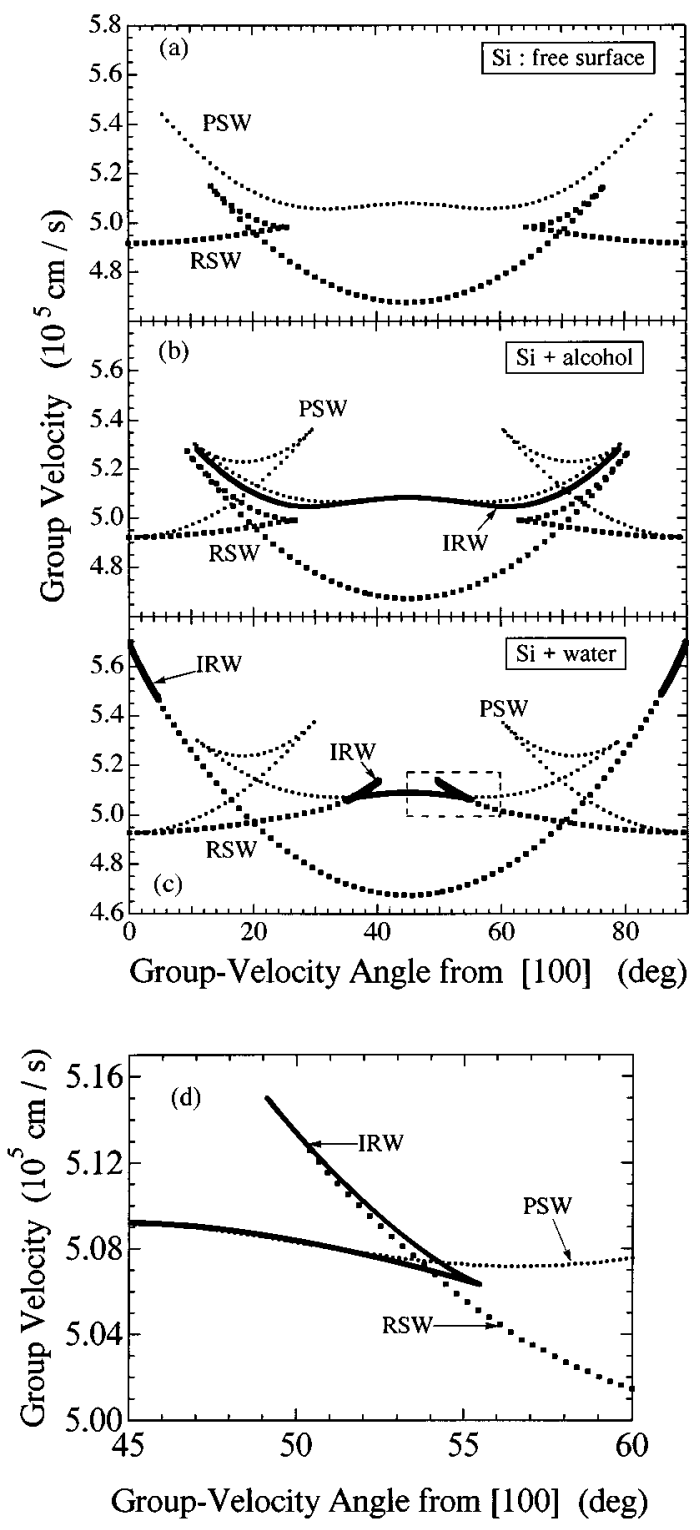

FIG. 3. Calculated group velocities of the RSW, PSW (dotted lines), and IRW (solid lines) on the (001) silicon substrate with (a) free surface, (b) alcohol loading, and (c) water loading. (d) The magnification of the structure enclosed by the dashed lines in (c). The abscissa is the angle in the real space measured from the [100] direction (not the angle of the wave vector). In part (c), the identifications of the RSW and IRW branches have been corrected from those in Ref. 8.

(001) surface of silicon shown in Fig. 3(a). ${ }^{16}$ For water loading [Fig. 3(c)], the cusps do not exist in the group-velocity curves of the RSW, but they instead appear in the IRW branch (at $34.5^{\circ}$ and $40.8^{\circ}$ from the [100] and their equivalent directions). Also, folds appear in the group-velocity curves of the PSW branch for both the alcohol and water loadings. Note that they do not exist in the PSW branch for the free (001) surface of silicon.

One of the remarkable features of the liquid loading predicted for the surface wave propagation is the fact that the group velocities of the RSW and IRW are very sensitive to the liquid loading, e.g., there is a continuous transition between the RSW and IRW branches for water loading, but discontinuities exist between these branches for alcohol load- 


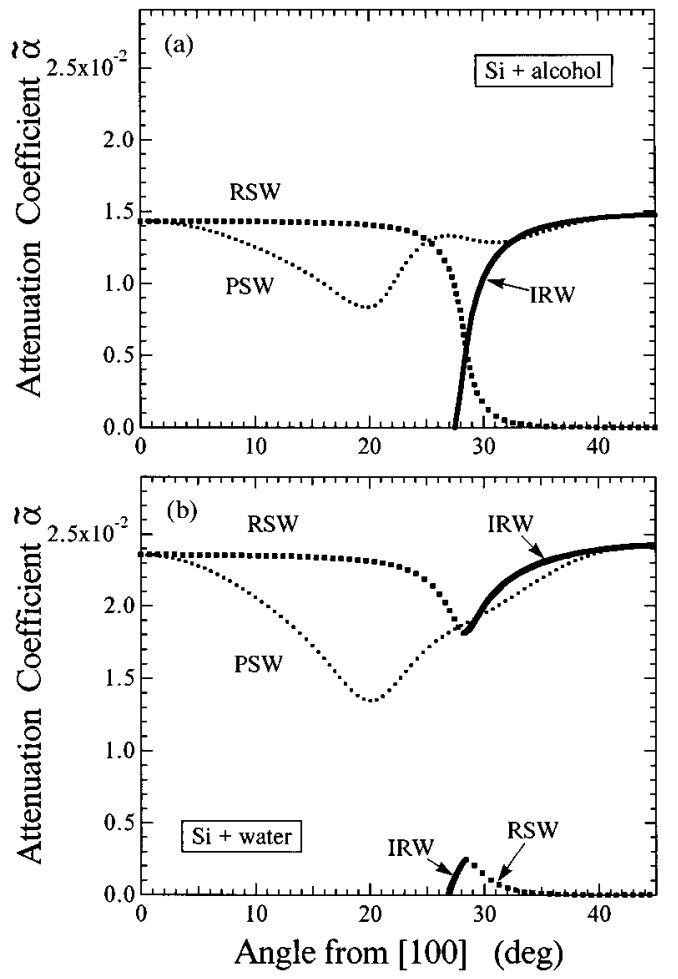

FIG. 4. Attenuation coefficients $\widetilde{\alpha}$ 's of the surface waves versus the propagation direction on the (001) plane of silicon, with (a) alcohol loading and (b) water loading. The abscissa is the wavevector angle measured from the [100] direction. The coefficients of the RSW and PSW are plotted by dotted lines and those of IRW are plotted by solid lines.

ing. For water loading, the continuity of the RSW and IRW makes a " $V$ " structure in the [100] directions, which has been observed experimentally. ${ }^{8,9}$

In Fig. 4, the attenuation coefficients $\widetilde{\alpha}$ 's are plotted as a function of wave-vector direction. The overall magnitude is larger for the water loading than the alcohol loading, because $Z_{L} / Z_{S}=0.11$ for the former case and $Z_{L} / Z_{S}=0.068$ for the latter case, respectively. For the case of water loading, it is clearly seen that the RSW branch at lower angles connects smoothly to the IRW branch.

\section{ORIGINS OF EXTRA BRANCHES}

As previously discussed, the character of the surface waves (RSW or PSW) is determined by the form of the wave numbers $k_{3}$ that describe the wave motion in the solid perpendicular to the surface. From Eq. (6), it follows that $k_{3}$ is a double-valued function of the sound velocity and, in general, is complex. If $k_{3}$ has a positive imaginary part, the wave is localized near the solid surface, and if $k_{3}$ has a negative imaginary part, the wave amplitude increases in the bulk of the solid with the distance from the surface. Which of the two values of $k_{3}$ is required for a partial wave depends on the boundary conditions and also on the propagation direction.

To get an insight into this point, first we consider the free (001) surface of silicon and two specific propagation directions, i.e., the [100] and [110] directions. In these directions, the lattice vibrations polarized within the saggital plane (the saggital modes) are decoupled from those polarized perpendicular to the same plane (the shear modes with horizontal polarization, or SH modes). The Rayleigh wave solutions are found in the saggital mode, where the $L$ (longitudinal) and FT (fast transverse) waves are coupled and their wave numbers $k_{3}^{(1)}$ and $k_{3}^{(2)}$ are complex with positive imaginary parts $\left(\left[k_{3}^{(1)}\right]^{*}=-k_{3}^{(2)}\right)$. Their velocities are indicated by the points marked by $A$ and $B$ in Fig. 2(a).

As Table I illustrates, at points $A$ and $B$, only two $\left(k_{3}^{(1)}\right.$ and $k_{3}^{(2)}$ ) of the three possible wave vectors contribute to the surface-wave solution. Since both the contributing wave vectors have positive imaginary components, their amplitudes decrease as the waves propagate into the solids; these waves are, by definition, Rayleigh surface waves. As propagation directions rotate away from these high-symmetry directions, a third-partial wave with SH-like polarization couples to the solution; the form of this partial wave determines whether the surface wave is a RSW or a PSW.

An important observation is that in the [100] direction the velocity of the RSW [at point A of Fig. 2(a)] is slower than

TABLE I. Values and forms of complex $k_{3}^{(\alpha)}(\alpha=1,2,3)$ describing the variation of the surface-wave amplitudes normal to the surface for several propagation directions in the (001) face of silicon $\left[a, a^{\prime}, b\right.$, $b^{\prime}, c$, and $d$ are real and positive, and $e$ and $\epsilon$ are small positive numbers depending on the angle from the chosen directions ([100] and [110]) and loading effect]. ID gives the identification of the branch in Fig. 2.

\begin{tabular}{|c|c|c|c|c|c|c|}
\hline Direction & ID & $\begin{array}{c}\text { On axis } \\
\text { (free surface) }\end{array}$ & $\begin{array}{l}\text { On axis } \\
\text { weights }\end{array}$ & $\begin{array}{c}\text { Near axis } \\
\text { (free surface) }\end{array}$ & $\begin{array}{c}\text { On axis } \\
\text { (water loaded) }\end{array}$ & $\begin{array}{l}\text { Near axis } \\
\text { (loaded) }\end{array}$ \\
\hline [100] & A & $\begin{array}{c}0.480+0.456 i \\
-0.480+0.456 i \\
0.540 i\end{array}$ & $\begin{array}{c}\sqrt{1 / 2} \\
\sqrt{1 / 2} \\
0\end{array}$ & $\begin{array}{c}a+b i \\
-a+b i \\
c i\end{array}$ & $\begin{array}{c}0.439+0.469 i \\
-0.522+0.441 i \\
\mp 0.044 \pm 0.538 i\end{array}$ & $\begin{array}{c}a+b i \\
-a^{\prime}+b^{\prime} i \\
\mp \epsilon \pm c i\end{array}$ \\
\hline [110] & B & $\begin{array}{c}0.402+0.532 i \\
-0.402+0.532 i \\
0.341\end{array}$ & $\begin{array}{c}\sqrt{1 / 2} \\
\sqrt{1 / 2} \\
0\end{array}$ & $\begin{array}{c}a+b i \\
-a+b i \\
d-e i\end{array}$ & $\begin{array}{c}0.359+0.547 i \\
-0.446+0.514 i \\
\mp 0.349 \pm 0.044 i\end{array}$ & $\begin{array}{c}a+b i \\
-a^{\prime}+b^{\prime} i \\
\mp d \pm e i\end{array}$ \\
\hline [110] & $\mathrm{C}$ & $\begin{array}{c}0.419+0.619 i \\
-0.419+0.619 i \\
0\end{array}$ & $\begin{array}{l}0 \\
0 \\
1\end{array}$ & $\begin{array}{c}a+b i \\
-a+b i \\
e i\end{array}$ & $\begin{array}{c}0.419+0.619 i \\
-0.419+0.619 i \\
0\end{array}$ & $\begin{array}{c}a+b i \\
-a^{\prime}+b^{\prime} i \\
-\epsilon+e i\end{array}$ \\
\hline
\end{tabular}




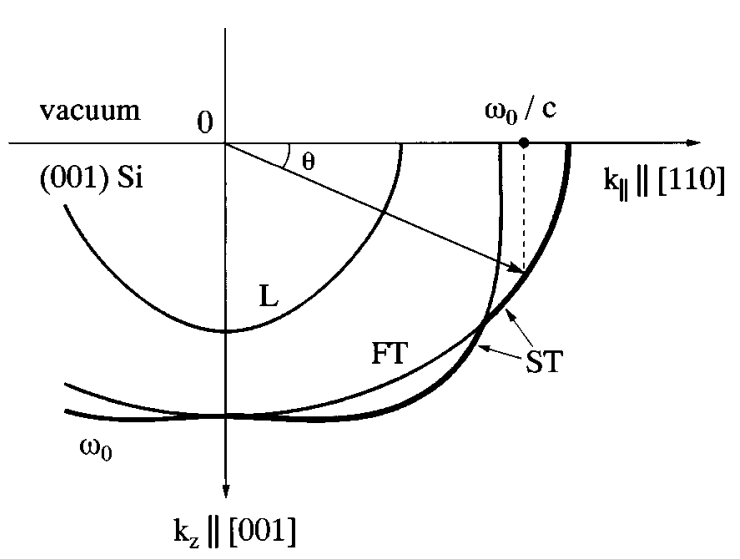

FIG. 5. The (110) section of the constant-frequency surfaces of the bulk elastic waves [longitudinal $(L)$, fast transverse (FT), and slow transverse $(\mathrm{ST})]$ in silicon. The dot indicates the location of the wave number $\left(k_{\|}=\omega_{0} / c, \omega_{0}\right.$ is a given frequency and $c=5.081 \times 10^{5} \mathrm{~cm} / \mathrm{s}$ ) of the true surface wave in the [110] direction (free surface). The corresponding location in Fig. 2(a) is point $B$ and this wave is on the PSW branch. For the propagation slightly away from the [110] direction, this wave couples to the bulk ST wave (the outermost curve), and hence radiates the energy into the bulk of the solid at the angle $\theta$ indicated $\left(\theta=23.1^{\circ}\right.$ in the [110] of silicon).

that of the bulk ST wave, but in the [110] direction the RSW [at point $B$ of Fig. 2(a)] is faster than the ST wave (see Figs. 1 and 5). Thus, the RSW solution near the [100] direction is stable even if this coupling turns on, i.e., the RSW does not decay into a bulk wave, because the velocity of the RSW in this direction is much slower than that of the bulk ST wave, leading to the RSW velocity change $\Delta c=c-c_{[100]}$ which is real, where $c_{[100]}$ is the velocity of the RSW in the [100] direction. (A complex velocity change is equivalent to a complex $k_{\|}$and signals a decay of the wave along the surface as shown in Sec. II.) In these directions, the wave number $k_{3}^{(3)}$ associated with the third partial wave coming from the coupling to the ST wave is pure imaginary (with positive imaginary part) and, thus, the wave is still localized near the surface.

Near the [110] direction, the situation is quite different. The ST branch (with SH character) has a velocity much slower than that of the true surface wave (RSW), embedded in the PSW branch at $45^{\circ}$. Accordingly, the coupling of the saggital and ST modes should lead to the decay of the waves in the PSW branch into the bulk transverse wave (Fig. 5). The analytical calculation specifically reveals that the velocity change near the [110] direction $\Delta c^{\prime}=c-c_{[110]}$ is complex, where $c_{[110]} \simeq 5.08 \times 10^{5} \mathrm{~cm} / \mathrm{s}$ is the phase velocity of the true surface wave in the [110] direction of $\mathrm{Si}$ (as explicitly shown in the Appendix for a fcc lattice). This indicates that the amplitude is attenuated as the wave propagates along the surface, which is characteristic of a PSW at a free surface. At the same time, the wave number $k_{3}^{(3)}$ acquires a small imaginary part near the [110] direction (in the [110] direction $k_{3}^{(3)}$ is real, but the partial wave characterized by $k_{3}^{(3)}$ is decoupled from the RSW), which must be negative to allow the damping of the wave along the surface, i.e., $k_{\|}$is complex, though $k_{3}^{(1)}$ and $k_{3}^{(2)}$ still have positive imaginary parts and represent the localized character (see Table I). Thus, away from the [110] direction the surface wave has a bulk-wave component, due to the coupling to the ST wave. However, the imaginary part of $k_{3}^{(3)} / k_{\|}^{(R)}$ is very small (typically $\left.10^{-4}-10^{-5}\right),{ }^{4,17}$ so the wave grows very slowly with the distance away from the surface and looks like a surface wave. This is the reason that the wave in this branch is called the PSW.

With a surface loading by a nonviscous liquid, RSW's on the free surface receive a perturbation and are no longer stable. They couple into the liquid, because their velocities are generally much higher than the sound velocity $c_{L}$ in the liquid. Thus, the amplitude of a RSW at the solid-liquid interface is attenuated as the wave propagates along the interface and radiates energy into the liquid, as shown in Fig. 1. In addition, the behavior of the wave amplitude inside solid is modified from the case of the free surface.

Quantitatively, the effect of the liquid loading is controlled by the ratio of the acoustic impedance of the liquid to that of the solid, which we denote by $a=Z_{L} / Z_{S}(\sim 0.1)$. For both the [100] and [110] directions the velocity changes, due to the liquid loading, are pure imaginary in the lowest order of $a$. (The finite correction to the velocity, which is real, arises in the order of $a^{2}$.) Owing to this attenuation of the lattice vibrations along the interface, double values of the wave number $k_{3}^{(3)}$ in a solid now become possible. As shown in Table I, this means that near the [100] direction, the PSW, which radiates energy into the bulk of the solid, appears in addition to the RSW . (The PSW is not supported on the free surface near the [100] direction, because there is no attenuation of the wave along the surface.)

The emission of waves into both the liquid and solid, i.e., $\operatorname{Im}[q]>0$ and $\operatorname{Im}\left[k_{3}^{(3)}\right]<0$, does not lead to any unphysical results if the wave is attenuated as it propagates along the interface. This can be seen by noting that

$$
\begin{aligned}
& \operatorname{Im}[q] z+\mathbf{k}_{\|}^{(I)} \cdot \mathbf{x}_{\|}=0 \quad(z<0), \\
& \operatorname{Im}\left[k_{3}^{(3)}\right] z+\mathbf{k}_{\|}^{(I)} \cdot \mathbf{x}_{\|}=0 \quad(z>0),
\end{aligned}
$$

hold for the wave emitted into the liquid $(z<0)$ and the third-partial wave emitted into the solid $(z>0)$, where $\mathbf{x}=\left(\mathbf{x}_{\|}, z\right)$ is a point along the group-velocity vector, or along the acoustic Poynting vectors of each wave (see Fig. 6). Hence, $\operatorname{Im}[q]>0$ and $\operatorname{Im}\left[k_{3}^{(3)}\right]<0$ satisfy Eqs. (15) and (16) if $k_{\|}^{(I)}>0$ holds. These equations also imply that the displacement amplitudes of the waves emitted from a point $O$ (on the interface) are constant along the lines in which the acoustic energy of the waves are propagated, i.e., $O P$ and $O Q$ in Fig. 6 which are parallel to the acoustic Poynting vectors $\mathbf{P}^{(L)}$ in the liquid and $\mathbf{P}^{(S)}$ in the solid defined in the next section. This is the consequence of the fact that we employ the linear elasticity theory without dissipation. It should be noted that in the liquid the direction of the energy flow is parallel to the wave vector $\mathbf{K}_{L}$ and only the thirdpartial wave governed by $k_{3}^{(3)}$ with $\operatorname{Im}\left[k_{3}^{(3)}\right]<0$ survives at a large distance $z$ in solid.

As the wave travels along the interface, its amplitude on the interface is attenuated exponentially, due to the emission of waves into the solid and liquid according to $\exp \left(-\mathbf{k}_{\|}^{(I)} \cdot \mathbf{x}_{\|}\right)$. Thus, on the plane perpendicular to the wave vector $\mathbf{k}_{\|}$, the waves emitted at a later time from the inter- 

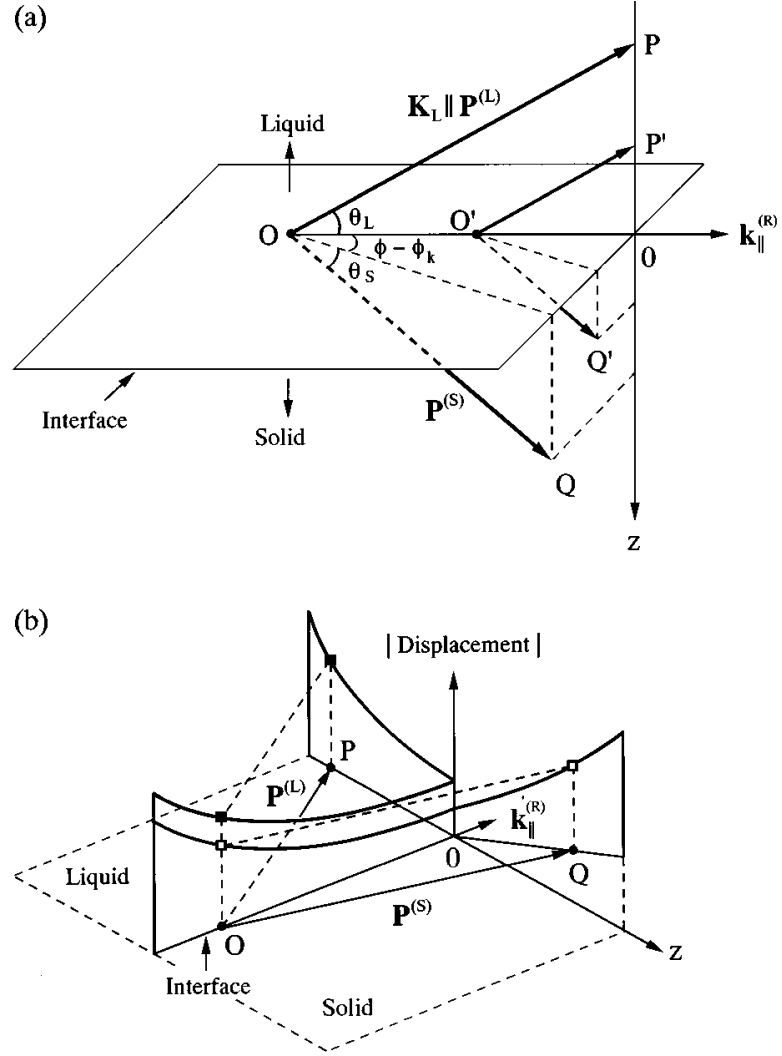

FIG. 6. (a) Schematic of the bulk wave radiation associated with the PSW. The waves are emitted from a point $\mathbf{O}\left(\mathbf{O}^{\prime}\right)$ on the interface into the liquid and solid sides along $O P\left(O^{\prime} P^{\prime}\right)$ and $O Q$ $\left(O^{\prime} Q^{\prime}\right)$, respectively. These lines are parallel to the acoustic Poynting vectors $\left(\mathbf{P}^{(L)}\right.$ and $\mathbf{P}^{(S)}$ ), or group velocity vectors of the longitudinal wave in liquid and the third partial wave (at a large $z$ ) in the solid, respectively. The displacement amplitudes are constant along these lines $O P\left(O^{\prime} P^{\prime}\right)$ in the liquid and $O Q\left(O^{\prime} Q^{\prime}\right)$ in the solid. The angles shown satisfy $\tan \theta_{L}=\operatorname{Im}[q] / k_{\|}^{(I)}$ and $\operatorname{Im}$ $\left[k_{3}^{(3)}\right] \tan \theta_{S}+k_{\|}^{(I)} \cos \left(\phi-\phi_{k}\right)=0$, where $\phi$ and $\phi_{k}$ are the azimuthal angles of the vectors $\mathbf{P}^{(S)}$ and $\mathbf{k}_{\|}$, respectively. (b) Schematic of the amplitude profiles of the PSW on the solid-liquid interface and also on the $z$ axis. The magnitudes of the lattice displacements at the points $\mathrm{O}$ and $\mathrm{P}$ associated with the longitudinal wave in liquid (filled squares) and at the points $\mathrm{O}$ and $\mathrm{Q}$ associated with the third partial wave in solid (open squares) are the same, leading to the exponential growths of the lattice displacement with the distances $|z|$ from the interface.

face (e.g., from a point $O^{\prime}$ ) are located closer to the interface $(z=0)$, i.e., at points $P^{\prime}$ and $Q^{\prime}$, and their amplitudes are much smaller than the amplitudes of the waves at points $P$ and $Q$ emitted earlier. This leads to the fact that the profile of the wave amplitude grows exponentially as the distance $|z|$ from the interface increases on both the solid and liquid sides [Fig. 6(b)].

Near the [110] direction, on the other hand, the RSW-type wave localized in the vicinity of the interface [all the associated $k_{3}^{(\alpha)}(\alpha=1,2,3)$ have positive imaginary parts] is induced by the liquid loading. For this mode (IRW), there exists no emission of wave into the solid side and the attenuation of the wave amplitude along the interface is balanced with the emission of the wave only into the liquid. All these features shown above are confirmed not only by numerical calculations for silicon, but also by the analytical calculations using the elastic constants of an anisotropic fcc lattice, i.e., $C_{11}=2 C_{12}=2 C_{44} \cdot{ }^{20}$ The details of these calculations are given in the Appendix.

There is a third location where the SAW takes on unusual behavior. At the point labeled $C$ for [110] propagation [Fig. 2(a)], solving the Christoffel equations reveals - as at points $A$ and $B$ - two partial waves with wave numbers, $k_{3}^{(\alpha)}(\alpha=1$ and 2$)$, are polarized in the saggital plane and one that with $k_{3}^{(3)}$ is not. Unlike the previous case, however, the first two-partial waves do not contribute to the SAW solution. Also unexpectedly, $k_{3}^{(3)}=0$. This solution (solution $C$ in Table I) is, in fact, the bulk ST wave polarized horizontally (SH wave); in this one direction, this bulk mode happens to satisfy the zero-stress boundary conditions at a free surface. As the propagation direction is rotated away from [110], the bulk ST wave does not satisfy these boundary conditions and the coupled $L$ and FT modes begin to contribute to forming the surface wave. In addition, the wave number $k_{3}^{(3)}$ corresponding to the SH-like partial wave, becomes pure imaginary (with positive imaginary part), although quite small, indicating a slow decrease in wave amplitude as the wave enters the solid. ${ }^{4}$ Thus, our bulk ST wave at [110] becomes a Rayleigh surface wave near [110] to satisfy the stress-free boundary condition. While the addition of surface loading allows both a RSW and a PSW to emerge from points $A$ and $B$, relaxing the boundary conditions near $C$ does not allow a PSW solution near this point. This is because in this direction a SH-like partial wave, with amplitude growing into the solid, already contributes to the upper PSW branch as discussed above.

\section{ENERGY FLOW}

Because the penetration distance of the amplitude of an RSW into solid is finite, it is capable of radiating acoustic energy only into liquid as the wave propagates along the solid-liquid interface. How about the PSW? An interesting issue is whether a PSW radiates energy predominantly into the solid or both into solid and liquid comparably. To answer this question, we calculate the acoustic Poynting vectors associated with the RSW and PSW, defined by ${ }^{4}$

$$
P_{i}^{(m)}=-\frac{1}{2} \operatorname{Re}\left\{T_{i j}^{(m)}\left[\dot{W}_{j}^{(m)}\right]^{*}\right\} \quad(i=1,2,3),
$$

where $m=L, S$ stand for liquid and solid, respectively, and $T_{i j}^{(m)}$ is the stress tensor (note that $\Sigma_{j}=T_{3 j}^{(L)}$ and $\sigma_{j}=T_{3 j}^{(S)}$ [see Eqs. (4) and (7)]), and $\mathbf{W}^{(L)}=\mathbf{U}$ and $\mathbf{W}^{(S)}=\mathbf{u}$.

In order to define the energy emissivities of the RSW and PSW into solid and liquid we consider a cylindrical region of height $z_{1}+z_{2}$ and of radius $r$ of the top and bottom faces as shown in Fig. 7 and calculate the acoustic flux

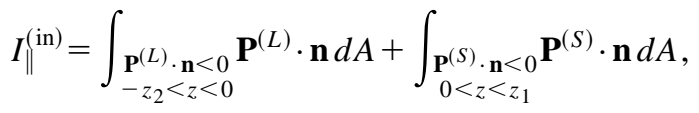

incident on this region through the sidewall, where $d A$ is a surface element, $\mathbf{n}$ is the unit vector normal to the sidewall and the integral should be taken over the area of the sidewall 


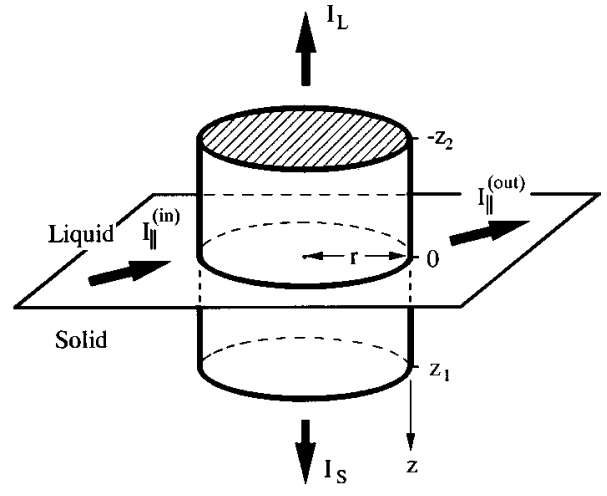

FIG. 7. Schematic of the cylindrical region assumed for the calculation of the acoustic flux and emissivities of the surface waves.

for which $\mathbf{P}^{(m)} \cdot \mathbf{n} \leqslant 0$. Similarly, the flux leaving from this region through the sidewall is

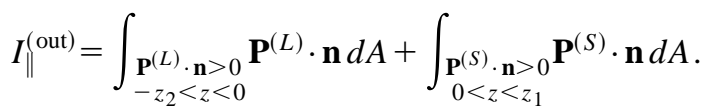

The flux emitted into the solid through the bottom face of the cylinder is

$$
I_{S}=\int_{z=z_{1}>0} P_{3}^{(S)} d A
$$

and that emitted into the liquid through the upper face of the cylinder is

$$
I_{L}=-\int_{z=-z_{2}<0} P_{3}^{(L)} d A .
$$

Now the sum $I_{\|}^{\text {(in) }}-I_{\|}^{\text {(out) }}-I_{S}+I_{L}$ should vanish, which guarantees the conservation of the acoustic energy. Thus, we can define the quantities, which measure the energy emissivities of acoustic waves into solid $\left(E_{S}\right)$ and into liquid $\left(E_{L}\right)$, as

$$
E_{S}=-I_{S} / I_{\|}^{(\mathrm{in})}, \quad E_{L}=I_{L} / I_{\|}^{(\mathrm{in})} .
$$

Of course $E_{S}$ and $E_{L}$ depend on the areas (or the radius $r$ ) of the top and bottom faces of the cylinder and also on $z_{1}$ and $z_{2}$. Figure 8 plots the emissivities $E_{L}$ into liquid (with $z_{1}=z_{2}=5 \lambda$ and $r=2 \lambda$, where $\lambda$ is the wavelength of the RSW in the [100] direction) of surface-related acoustic modes in (100) silicon for alcohol and water loadings. As expected, RSW emits the acoustic energy only into liquid though the emissivity vanishes in the [110] direction, where the RSW becomes an SH wave, which does not couple to the longitudinal wave in liquid. The IRW also emits the energy only into liquid. For propagations close to the angle where the IRW branch degenerates into the bulk ST wave (at angles $\sim 27^{\circ}$ ), the negative emissivity into the solid $\left(E_{S}<0\right)$ can be seen for both alcohol and water loadings. This means that at these propagation angles, the energy flow from the solid to liquid exists due to the bulklike nature of IRW branch.

More interesting results can be seen for the PSW branch. The energy emission into liquid associated with the PSW is vanishingly small at angles smaller than $\sim 25^{\circ}$ from the
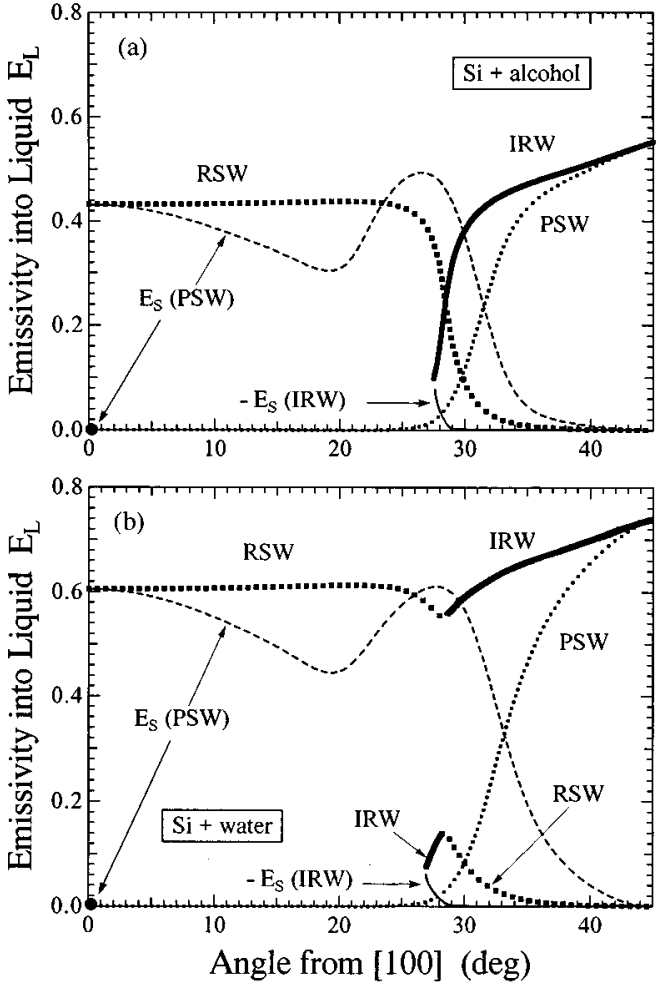

FIG. 8. Energy emissivities $E_{L}$ 's of the RSW, PSW (dotted lines), and IRW (solid lines) into liquid in the presence of (a) alcohol loading and (b) water loading. The abscissa is the wave-vector angle measured from the [100] direction. (The parameters chosen are $z_{1}=z_{2}=5 \lambda$ and $r=2 \lambda$, where $\lambda$ is the wavelength of the RSW in the [100] direction.) The emissivities $E_{S}$ 's of the PSW and IRW $\left(-E_{S}\right.$ for the IRW) into the solid are also plotted by dashed lines and thin solid lines, respectively. At $0^{\circ}$ ([100] direction) $E_{S}$ for the PSW vanishes.

[100] direction, i.e., the portion of the PSW branch induced by the liquid does not radiate acoustic vibrations into the liquid, but rather emits the energy into the bulk of solid. At angles larger than $\sim 25^{\circ}$, however, the energy emissivity of the PSW into the solid is suppressed and emission into liquid appears. ${ }^{21}$ These latter angles are those at which the PSW's exist for free surface and they behave rather like surface waves. ${ }^{17}$ Thus, the presence of induced PSW's at angles near the [100] direction cannot be observed by measuring energy transmission into liquid, but could be confirmed by observing the energy transmission into the solid.

It should be noted that in the [100] direction the induced PSW coincides with the RSW, so their energy emissivities into liquid should also coincide, i.e., $E_{L}=0$. Actually, $E_{L}$ for the PSW very sharply increases to the value of the RSW at angles close to $0^{\circ}$, which is, however, not explicitly seen in Fig. 8.

\section{CONCLUDING REMARKS}

In the present work, we studied theoretically the effects of liquid loading on the properties of the surface acoustic waves propagating on the (100) face of silicon. The liquid loading studied previously had been restricted to the case of isotropic elastic substrate and only the appearance of complex wave 
numbers (equivalently, the velocity change and attenuation of waves) perturbed by the loading was recognized. However, the presence of the elastic anisotropy produces a more interesting effect as discussed in this paper. Similar results are also expected for the liquid loading on the surfaces of lower symmetry, or on surfaces of crystals with noncubic symmetry. ${ }^{22}$

It should be noted that the extension of the PSW branch induced by the liquid loading predominantly emits the energy into the solid substrate, so the existence of this portion of the PSW branch cannot be confirmed by measuring the acoustic signal in the liquid. For its observation the acoustic signal propagating slower than the bulk ST mode, but faster than the RSW, must be detected in the solid side. This remains an interesting challenge.

We also remark here that the PSW's studied here are those with only one-partial wave, which grows into the bulk of the solid substrate (for instance, $\operatorname{Im}\left[k_{3}^{(1)}\right]>0$, $\operatorname{Im}$ $\left[k_{3}^{(2)}\right]>0$, but $\left.\operatorname{Im}\left[k_{3}^{(3)}\right]<0\right)$. Actually, the PSW solutions with two partial waves, the amplitudes of which grow into the solid also exist. ${ }^{22}$ However, these PSW's generally consist at least of one partial wave with a large growth rate into the solid and also a large attenuation coefficient $\widetilde{\alpha}$ along the solid-liquid interface. Hence, it should be hard to observe the associated acoustic energy emitted into liquid.

The frequencies of the SAW's excited experimentally with ultrasonic immersion transducers are typically in the range of $10 \mathrm{MHz}$ and the corresponding wavelengths are about $0.1 \mathrm{~mm}$. These lengths are not negligible compared to the propagation distances of the SAW's in the experiments. Thus, the finite-wavelength effects such as diffraction and interference should play important roles in the focusing experiments using coherent SAW's in the above frequency range. The similar effects have already been observed in the focusing experiments of bulk acoustic waves of $10-\mathrm{MHz}$ range. ${ }^{23}$ We plan to analyze the group velocities of the SAW's at solid-liquid interfaces taking account of the finitewavelength effects.

\section{ACKNOWLEDGMENTS}

This work was supported in part by the Murata Science Foundation, and by the National Science Foundation under Materials Research Laboratory Grant No. 89-20538.

\section{APPENDIX: FCC MODEL CALCULATIONS}

To understand the origin of the PSW and the IRW, we consider the surface wave solutions near the [110] direction on the (001) surface of a cubic crystal. The elastic constants in the fcc lattice, i.e., $C_{11}=2 C_{12}=2 C_{44},{ }^{20}$ are used as an illustration. Here, we note that the anisotropy factor $A=2 C_{44} /\left(C_{11}-C_{12}\right)=2$ in the fcc lattice leads to qualitatively the same angular dependence of the sound velocities of surface and bulk waves as in silicon $(A=1.57)$, germanium $(A=1.67)$ and $\mathrm{GaAs}(A=1.83)$.

Figure 9 plots the normalized phase velocities versus propagation direction of several acoustic modes on the (001) face of the fcc lattice. Bulk transverse modes satisfy the stress-free boundary condition at the points marked by dots [also at these points bulk slow transverse (ST) waves become

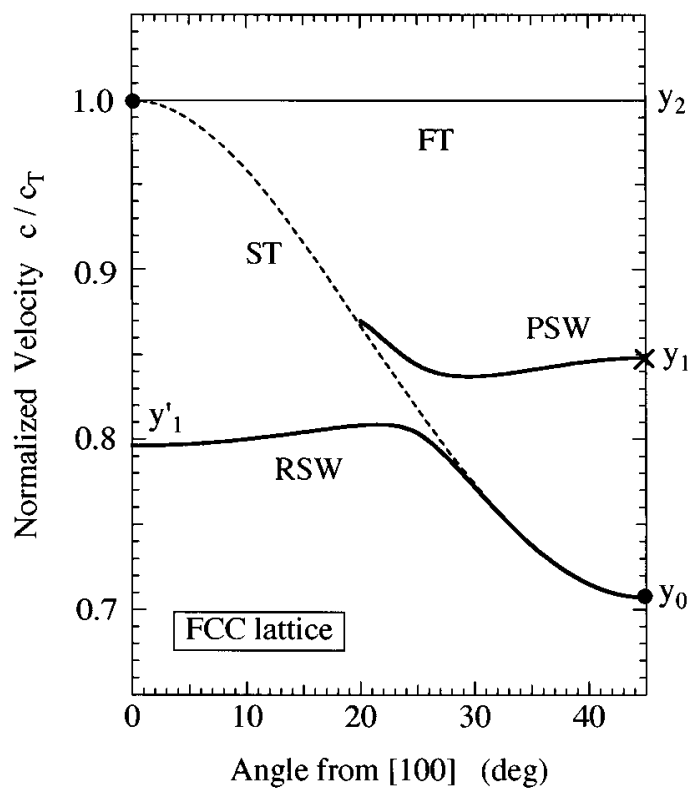

FIG. 9. Normalized velocities $\left(y=c / c_{t}\right)$ of the bulk transverse and surface acoustic waves propagating in the (001) plane of the fcc lattice (the surface is stress-free for the RSW and PSW, and the abscissa is the wave-vector angle measured from the [100] direction). $\quad y_{2}=1, \quad y_{1}^{2}=(7-\sqrt{17}) / 4=(0.848)^{2}, \quad\left(y_{1}^{\prime}\right)^{2}=(3-\sqrt{3}) / 2$ $=(0.796)^{2}$, and $y_{0}^{2}=1 / 2$. The cross indicates the velocity of the true surface wave in the [110] direction. At the points marked by dots, the waves are polarized perpendicular to the saggital plane, i.e., $\mathrm{SH}$ waves.

SH waves polarized perpendicular to the saggital plane]. The velocity of the RSW degenerates into a bulk ST wave in the [110] direction ( $y_{0}$ in Fig. 9) and in this direction the true surface wave localized near the surface appears in the PSW branch with the normalized velocity $y_{1}$ (the cross in Fig. 9). Except for the [110] direction, the wave in the PSW branch has a partial wave, the amplitude of which grows with the distance from the surface and becomes degenerate with the bulk ST mode at angles smaller than $\sim 20^{\circ}$.

\section{1. [110] propagation: free surface}

In this case, the saggital modes (longitudinal and FT waves) are decoupled from the SH mode (ST wave) and the equations of motion become

$$
\left(\begin{array}{cc}
5 k_{\|}^{2} / 2+k_{3}^{2}-\rho_{S} \omega^{2} / C_{44} & 2 k_{\|} k_{3} \\
2 k_{\|} k_{3} & k_{\|}^{2}+2 k_{3}^{2}-\rho_{S} \omega^{2} / C_{44}
\end{array}\right)\left(\begin{array}{c}
\varepsilon_{\|} \\
\varepsilon_{3}
\end{array}\right)=0,
$$

for the saggital mode and

$$
\left(k_{\|}^{2} / 2+k_{3}^{2}-\rho_{S} \omega^{2} / C_{44}\right) \varepsilon_{\perp}=0,
$$

for the SH mode. In these equations, $k_{\|}$is the wave number in the [110] direction [within the (001) surface] and $k_{3}$ is the component perpendicular to the surface (the solid occupies the half space $x_{3}>0$ ).

The secular equation obtained from Eq. (A1) determines the wave number $k_{3}$ of the saggital mode for a given frequency $\omega=k_{\|}$. Now we introduce the variables, 
$\kappa=k_{3} / k_{\|}, y^{2}=c^{2} / c_{t}^{2}\left[c_{t}=\left(C_{44} / \rho_{S}\right)^{1 / 2}\right.$ is the velocity of the isotropic FT mode in the (001) plane], and $z=\kappa^{2}-y^{2}+1 / 2$. The secular equation corresponding to Eq. (A1) reads

$$
2 z^{2}+y^{2} z-2\left(y^{2}-1\right)=0 .
$$

Thus, the saggital modes have the solutions

$$
z=\frac{1}{4}\left\{-y^{2} \pm\left[y^{4}+16 y^{2}-16\right]^{1 / 2}\right\} \equiv\left(\begin{array}{c}
z^{(1)} \\
z^{(2)}
\end{array}\right),
$$

or

$$
\left(\begin{array}{c}
{\left[\kappa^{(1)}\right]^{2}} \\
{\left[\kappa^{(2)}\right]^{2}}
\end{array}\right)=\frac{1}{4}\left(3 y^{2}-2 \pm\left[y^{4}+16 y^{2}-16\right]^{1 / 2}\right) .
$$

Also Eq. (A2) gives for the SH mode

$$
z=0 \equiv z^{(3)}
$$

or

$$
\left[\kappa^{(3)}\right]^{2}=y^{2}-1 / 2 .
$$

The polarization vectors of the saggital modes corresponding to $z^{(1)}$ and $z^{(2)}$ are determined from Eq. (A1) or

$$
\left(\begin{array}{cc}
2+z^{(i)} & 2 \kappa^{(i)} \\
2 \kappa^{(i)} & 2 z^{(i)}+y^{2}
\end{array}\right)\left(\begin{array}{c}
\varepsilon_{\|}^{(i)} \\
\varepsilon_{3}^{(i)}
\end{array}\right)=0 \quad(i=1,2) .
$$

In the above equations, the velocity $c$ (or normalized velocity $y$ ) is still unknown and is determined by the boundary conditions.

The stress-free boundary condition at the solid surface is written as

$$
\left|\begin{array}{cc}
\varepsilon_{3}^{(1)}+\kappa^{(1)} \varepsilon_{\|}^{(1)} & \varepsilon_{3}^{(2)}+\kappa^{(2)} \varepsilon_{\|}^{(2)} \\
\varepsilon_{\|}^{(1)}+2 \kappa^{(1)} \varepsilon_{3}^{(1)} & \varepsilon_{\|}^{(2)}+2 \kappa^{(2)} \varepsilon_{3}^{(2)}
\end{array}\right|=0 .
$$

This leads to

$$
2 y^{6}-15 y^{4}+32 y^{2}-16=\left(y^{2}-4\right)\left(2 y^{4}-7 y^{2}+4\right)=0 .
$$

Hence, we find

$$
y^{2}=c^{2} / c_{t}^{2}=4, \quad(7 \pm \sqrt{17}) / 4
$$

Among these solutions, $y=y_{1}=c_{1} / c_{t}=[7-\sqrt{17}]^{1 / 2} / 2$ $=0.848$ (the cross in the [110] direction of Fig. 9) corresponds to the surface-wave velocity in the [110] direction. This velocity is larger than the velocity of the ST mode $c_{\mathrm{st}}=c_{t} / \sqrt{2}$ or $y_{0}=c_{\mathrm{st}} / c_{t}=1 / \sqrt{2}$ in the [110] direction (Fig. 9). Substituting $y=y_{1}=0.848$ into Eq. (A5), we have $\left(\kappa^{(1)}, \kappa^{(2)}\right)= \pm 0.519+0.480 i$ for the saggital mode. These constants govern the decay of the wave amplitudes into the solid. For this velocity $\kappa^{(3)}=\sqrt{y_{1}^{2}-1 / 2}=0.648$. This is real (because $y_{1}>y_{0}$ or $c_{1}>c_{\text {st }}$ ) and the wave is a bulk SH mode. The associated $\mathrm{SH}$ wave is, however, decoupled from the saggital mode in the [110] direction. At an angle close to the [110] direction, the SH-like partial wave characterized by $\kappa^{(3)}$ couples to the saggital mode. This coupling causes the attenuation of the surface-wave amplitudes with propagation distance as shown below.

\section{A propagation close to the [110] axis: free surface}

For a propagation angle $\theta=\pi / 4-\delta(\delta \ll 1)$ measured from the [100] axis on the (001) surface, the equation of motion (6) becomes

$$
\left(\begin{array}{ccc}
2(2+z)-\delta^{2}(4+z), & -2 \delta(1+z)+\delta^{3}(z+7) / 3 & 2\left(2-\delta^{2}\right) \kappa \\
2(1+z)+\delta^{2}(5-z) / 3, & 2 z+\delta^{2}(2+z), & 4\left(\delta-\delta^{3} / 6\right) \kappa \\
2 \kappa & 0 & 2 z+y^{2}
\end{array}\right)\left(\begin{array}{c}
\varepsilon_{\|} \\
\varepsilon_{\perp} \\
\varepsilon_{3}
\end{array}\right)=0
$$

where $z=\kappa^{2}-y^{2}+1 / 2$ as before. From this equation we find, up to the order of $\delta^{2}$,

$$
\begin{gathered}
{\left[\kappa^{(1)}\right]^{2}=y^{2}-1 / 2+\beta+\delta^{2} \xi / 2, \quad \xi=\left(2 \beta+5 y^{2}-4\right) /[\beta(\beta-\gamma)],} \\
{\left[\kappa^{(2)}\right]^{2}=y^{2}-1 / 2+\gamma+\delta^{2} \zeta / 2, \quad \zeta=\left(2 \gamma+5 y^{2}-4\right) /[\gamma(\gamma-\beta)],} \\
{\left[\kappa^{(3)}\right]^{2}=y^{2}-1 / 2+\delta^{2} \eta / 2, \quad \eta=\left(5 y^{2}-4\right) /\left(1-y^{2}\right),}
\end{gathered}
$$

where $\beta=z^{(1)}, \gamma=z^{(2)}$. Also the polarization vectors corresponding to $\kappa^{(1)}, \kappa^{(2)}$, and $\kappa^{(3)}$ are

$$
\begin{aligned}
& \left(\varepsilon_{\|}^{(1)}, \varepsilon_{\perp}^{(1)}, \varepsilon_{3}^{(1)}\right)=N^{(1)}\left[2\left(1-y^{2}\right)+\delta^{2} f^{(1)}, 2 \gamma \delta, 2 \beta \kappa^{(1)}\right], \\
& \left(\varepsilon_{\|}^{(2)}, \varepsilon_{\perp}^{(2)}, \varepsilon_{3}^{(2)}\right)=N^{(2)}\left[2\left(1-y^{2}\right)+\delta^{2} f^{(2)}, 2 \beta \gamma, 2 \gamma \kappa^{(2)}\right], \\
& \left(\varepsilon_{\|}^{(3)}, \varepsilon_{\perp}^{(3)}, \varepsilon_{3}^{(3)}\right)=N^{(3)}\left[y^{2} \delta, 2\left(1-y^{2}\right)+\delta^{2} f^{(3)}, 2 \kappa^{(3)} \delta\right],
\end{aligned}
$$

where $N^{(1)}, \quad N^{(2)}$, and $N^{(3)}$ are the normalization factors, and $f^{(1)}=-\beta \xi, \quad f^{(2)}=-\gamma \zeta, \quad$ and $f^{(3)}=\left(6 y^{4}-31 y^{2}+22\right) / 3\left(3 y^{2}-2\right)$.

Now the boundary condition determinant (13) reads 


$$
\left|\begin{array}{ccc}
\varepsilon_{3}^{(1)}+\kappa^{(1)} \varepsilon_{\|}^{(1)} & \varepsilon_{3}^{(2)}+\kappa^{(2)} \varepsilon_{\|}^{(2)} & \varepsilon_{3}^{(3)}+\kappa^{(3)} \varepsilon_{\|}^{(3)} \\
\kappa^{(1)} \varepsilon_{\perp}^{(1)} & \kappa^{(2)} \varepsilon_{\perp}^{(2)} & \kappa^{(3)} \varepsilon_{\perp}^{(3)} \\
\varepsilon_{\|}^{(1)}+2 \kappa^{(1)} \varepsilon_{3}^{(1)} & \varepsilon_{\|}^{(2)}+2 \kappa^{(2)} \varepsilon_{3}^{(2)} & \varepsilon_{\|}^{(3)}+2 \kappa^{(3)} \varepsilon_{3}^{(3)}
\end{array}\right|=0,
$$

where the $(1,1),(2,2)$, and $(3,3)$ elements are $O(1)$ and other elements are $O(\delta)$ [see Eq. (A14)]. This leads to the equation determining the normalized velocity $y$

$$
\begin{gathered}
4\left(1-y^{2}\right)^{2} \kappa^{(3)}\left(\kappa^{(1)}-\kappa^{(2)}\right)\left(1-y^{2}-2 y^{2} \kappa^{(1)} \kappa^{(2)}-4\left[\kappa^{(1)} \kappa^{(2)}\right]^{2}\right) \\
-\delta^{2}\left\{2\left(1-y^{2}\right) f^{(3)} \kappa^{(3)}\left(\kappa^{(1)}-\kappa^{(2)}\right)\left(1-y^{2}-2 y^{2} \kappa^{(1)} \kappa^{(2)}-4\left[\kappa^{(1)} \kappa^{(2)}\right]^{2}\right)\right. \\
+4\left(1-y^{2}\right)^{2} \kappa^{(3)}\left[\left(1-2 \kappa^{(1)} \kappa^{(2)}\right)\left(\zeta \kappa^{(1)}-\xi \kappa^{(2)}\right)+2\left(\kappa^{(1)}-\kappa^{(2)}\right)\right] \\
+\left(1-y^{2}\right)\left(2-y^{2}\right) \kappa^{(3)}\left(\kappa^{(1)}-\kappa^{(2)}\right)\left[2 y^{4}-y^{2}-2+2\left(y^{2}-2\right) \kappa^{(1)} \kappa^{(2)}\right] \\
\left.+2\left(3 y^{2}-2\right)^{2}\left(\left[\kappa^{(1)}\right]^{2}-\left[\kappa^{(2)}\right]^{2}\right) \kappa^{(1)} \kappa^{(2)}\right\}=0 .
\end{gathered}
$$

Note that we have to keep the terms of $O\left(\delta^{2}\right)$ in the first term of Eq. (A16) [the terms of $O\left(\delta^{2}\right)$ are contained in $\left.\kappa^{(\alpha)}\right]$, but we may put $\delta^{2}=0$ inside the curly bracket. Thus, we can write the complicated Eq. (A16) in the form

$$
g\left(y, \delta^{2}\right)-\delta^{2} h(y)=0,
$$

where $g$ is the first term of Eq. (A16) and $h$ is the term inside the curly bracket of Eq. (A16). Putting $y=y_{1}+\Delta y$, where $\Delta y=O\left(\delta^{2}\right)$, and using the equation $g\left(y_{1}, 0\right)=0$ (this gives the normalized frequency $y_{1}$ in the [110] direction), we have

$$
\Delta y=\delta^{2}\left[h\left(y_{1}\right)-\partial g\left(y_{1}, \delta^{2}\right) /\left.\partial \delta^{2}\right|_{\delta^{2}=0}\right] /\left[\partial g(y, 0) /\left.\partial y\right|_{y_{1}}\right] .
$$

Here, we note that for $\delta=0$, i.e., for the [110] propagation, the wave numbers $\kappa^{(1)}$ and $\kappa^{(2)}$ are complex numbers of the forms $\kappa^{(1)}\left(y_{1}\right)=a+b i, \quad \kappa^{(2)}\left(y_{1}\right)=-a+b i$, and $\kappa^{(3)}\left(y_{1}\right)=d$, where $a, b$, and $d$ are positive real numbers, as shown above (see Table I). This means that $\kappa^{(1)}+\kappa^{(2)}$ is pure imaginary, but $\kappa^{(1)}-\kappa^{(2)}$ and $\kappa^{(1)} \kappa^{(2)}$ are both real, leading to the fact that $h\left(y_{1}\right)$ is complex [the last term of Eq. (A16) is pure imaginary] though $\partial g / \partial \delta^{2}$ and $\partial g / \partial y$ are real. Thus, we conclude that $\Delta y$ is a complex quantity, or at an angle in the vicinity of the [110] direction the normalized velocity $y=c / c_{t}=k_{t} / k_{\|}$becomes complex, i.e., the phonons in the branch that we are discussing attenuate as they propagate at an angle close to the [110] direction.

Because $y$ is now complex, the wave numbers $\kappa^{(3)}= \pm\left(y^{2}-1 / 2+\delta^{2} \eta / 2\right)^{1 / 2}$ are also complex. This implies that the small SH-like component, which couples (through $\left.\kappa^{(3)}\right)$ to the saggital mode near the [110] direction, also has a complex wave number perpendicular to the surface. The imaginary part of $\kappa^{(3)}$ must be negative so that the amplitude may grow exponentially with a distance from the surface. Thus, the wave of this mode is called a pseudosurface wave (PSW). The positive imaginary part of $\kappa^{(3)}$ (corresponding to a wave localized near the surface) is not allowed because of the attenuation of the wave along the surface.

For $\delta \neq 0$, the true surface wave appears below the ST branch at a velocity given by $y=y_{0}+\Delta y$, where $y_{0}=1 / \sqrt{2}$ $\left(c<c_{\mathrm{st}}=c_{t} / \sqrt{2}\right)$ and $\Delta\left(y^{2}\right)=-\delta^{2} \eta / 2=3 \delta^{2} / 2>0$. However, for this mode $\Delta \kappa^{(3)}=O\left(\delta^{4}\right)$, so we cannot say if $\kappa^{(3)}$ is pure imaginary (as expected), at least not in the approximation that we are employing. The reason for the decay of the PSW branch, except in the [110] direction, is the coupling to a bulk ST mode, which has slower sound velocity, i.e., $c_{\text {st }}<c_{1}$, as has been explained in Sec. IV (Fig. 5).

\section{3. $[110]$ propagation: with liquid loading}

When the solid surface is in contact with liquid, the equation determining the surface wave velocity [Eq. (10)] becomes, for the [110] propagation,

$$
\begin{gathered}
8 \kappa^{(1)} \kappa^{(2)}-\left(2\left[\kappa^{(1)}\right]^{2}+1-y^{2}\right)\left(2\left[\kappa^{(2)}\right]^{2}+1-y^{2}\right) \\
+2\left(2 \kappa^{(1)} \kappa^{(2)}-1\right)\left(2 \kappa^{(1)} \kappa^{(2)}-1+y^{2}\right) \\
=4 a y \kappa^{(1)} \kappa^{(2)}\left(\kappa^{(1)}+\kappa^{(2)}\right) / \kappa
\end{gathered}
$$

where $\quad a=Z_{L} / Z_{S}=\rho_{L} c_{L} / \rho_{S} c_{t} \quad(a \simeq 0.1) \quad$ and $\kappa \equiv\left(1-c_{L}^{2} / c^{2}\right)^{1 / 2}$. Using the equations

$$
\begin{gathered}
{\left[\boldsymbol{\kappa}^{(1)} \kappa^{(2)}\right]^{2}=\left(2 y^{4}-7 y^{2}+5\right) / 4,} \\
{\left[\kappa^{(1)}\right]^{2}+\left[\kappa^{(2)}\right]^{2}=\left(3 y^{2}-2\right) / 2,}
\end{gathered}
$$

obtained from Eq. (A5), we have

$$
y^{2} \kappa^{(1)} \kappa^{(2)}+y^{4}-3 y^{2}+2=a y \kappa^{(1)} \kappa^{(2)}\left(\kappa^{(1)}+\kappa^{(2)}\right) / \kappa .
$$

Setting $y=y_{1}+\Delta y$ and $\kappa^{(1,2)}=\kappa^{(1,2)}\left(y_{1}\right)+\Delta \kappa^{(1,2)}$, where $\Delta y, \Delta \kappa^{(1,2)}=O(a)$, and using the relation

$$
\Delta\left[\boldsymbol{\kappa}^{(1)} \boldsymbol{\kappa}^{(2)}\right]=\left(y^{3}-7 y / 4\right) \Delta y /\left[\boldsymbol{\kappa}^{(1)} \boldsymbol{\kappa}^{(2)}\right],
$$

we find

$$
\Delta y=\left.\frac{a \kappa^{(1)} \kappa^{(2)}\left(\kappa^{(1)}+\kappa^{(2)}\right)}{2 \kappa^{(1)} \kappa^{(2)}+4 y^{2}-6+\frac{y^{4}-7 y^{2} / 4}{\kappa^{(1)} \kappa^{(2)}}} \frac{1}{\kappa}\right|_{y=y_{1}} .
$$


Again $\kappa^{(1)} \kappa^{(2)}$ is real and $\kappa^{(1)}+\kappa^{(2)}$ is pure imaginary at $y=y_{1}$. Thus, $\Delta y$ is pure imaginary, which means that the attenuation of the surface wave is caused by liquid loading and its magnitude is of the order of $a$, but the velocity change (which should be a real quantity) is of the order of $a^{2}$. The latter has been checked by analytically calculating $\Delta y$ up to $O\left(a^{2}\right)$, although here we do not give the explicit formula, which is lengthy. Instead, we show that these results are in accord with the numerical calculations. According to Eq. (A23) and the analytical calculation up to $O\left(a^{2}\right)$ that we find for the water/silicon system, $\quad \operatorname{Im}\left[\Delta k_{\|}\right] / k_{\|}=i \Delta y / y_{1}=0.0244 \quad(0.0243) \quad$ and $\operatorname{Re}\left[\Delta k_{\|}\right] / k_{\|}=\Delta c / c_{1}=0.00219$ (0.00216), which should be compared with the numerically obtained attenuation and velocity change shown in the parentheses.

\section{A propagation close to the [110] axis: with liquid loading}

In this case, the equation determining the velocity $y$ is Eq. (A16), but the r.h.s. is finite with a contribution proportional to $a$ [similar to the r.h.s. of Eq. (A19)] coming from the liquid loading. We note that the terms, except for the last one of the 1.h.s. of Eq. (A16), are proportional to $\kappa^{(3)}$, which is double valued. $\left(\kappa^{(1)}\right.$ and $\kappa^{(2)}$ are also double valued, but their signs have been chosen so that the wave may decay with the distance from the solid surface.) Without liquid loading, one of those two values (with different signs) of $\kappa^{(3)}$ is not allowed in the branch starting from $y_{1}$ (PSW), because $y$ is complex (irrespective of the sign of $\kappa^{(3)}$ ) and the wave must decay along the surface. $\kappa^{(3)}$ with the positive imaginary part (though very small $10^{-3}-10^{-5}$ ) corresponds to the wave localized near the surface, so this is not accommodated to the attenuation of the waves along the surface.

However, with liquid loading both values of $\kappa^{(3)}$ become possible, because the wave is always attenuated along the surface by the continuous transmission of the energy into the liquid (i.e., complex wave number $q$ in the liquid side perpendicular to the interface [see Eq. (2)]). So, the energy transmission into the solid is not necessary for the attenuation of the wave along the surface. Thus, one of $\kappa^{(3)}$ 's with a positive imaginary part (localized wave near the solid surface) is now allowed, leading to the liquid induced Rayleigh wave (IRW). It is important to note that $\operatorname{Im}\left[\kappa^{(3)}\right]=O(a)$, i.e., the decay constant is of the order of 0.1 or less $\left(\operatorname{Im}\left[\kappa^{(3)}\right]= \pm 0.044\right.$ in the [110] for the water/silicon system).

Here, we compare this situation with that in a direction close to the [100], where no PSW solution exists for the free surface. For this direction $\left[\kappa^{(3)}\right]^{2}=y^{2}-1+O\left(\delta^{2}\right)$, where $\delta$ is, this time, a small angle measured from the [100] axis. The fcc model calculation for the free surface gives $y=y_{1}^{\prime}=[(3-\sqrt{3}) / 2]^{1 / 2}=0.796<1$ in the [100] (see Fig. 9). So, $\kappa^{(3)}$ is pure imaginary for $y=y_{1}^{\prime}$ and its magnitude is of the order of $0.5\left(\kappa^{(3)}= \pm 0.605 i\right)$. This leads to the fact that the correction to $y_{1}^{\prime}$ for a finite $\delta$ [derived from an equation quite similar to Eq. (A16) $]^{24}$ near the [100] is real. Thus, there is no attenuation along the surface and the surface wave keeps its localized character, i.e., only a RSW is allowed. $\left[\kappa^{(3)}\right.$ with negative imaginary part (PSW) is not allowed as a solution because there is no attenuation along the surface.] With liquid loading, the attenuation of the wave along the surface is induced, as in the case of [110] propagation. Thus, $y$ becomes complex and its imaginary part is $O(a)$. This means that $\left[\kappa^{(3)}\right]^{2}$ becomes complex and both $\kappa^{(3)}$ 's with positive and negative imaginary parts are now possible, leading to the appearance of the PSW. Note that near the [100] direction, the IRW coincides with the RSW.
${ }^{1}$ Lord Rayleigh, Proc. London Math. Soc. 17, 4 (1887).

${ }^{2}$ See, for example, Acoustic Surface Waves, edited by A. A. Oliner (Springer, New York, 1978).

${ }^{3}$ A. A. Maradudin, in Nonequilibrium Phonon Dynamics, edited by W. E. Bron (Plenum, New York, 1985), Chap. 10.

${ }^{4}$ G. W. Farnell, in Physical Acoustics VI, edited by W. P. Mason and R. N. Thurston (Academic, New York, 1970), p. 109.

${ }^{5}$ B. D. Rouhani, L. Dobrzynski, O. H. Duparc, R. E. Camley, and A. A. Maradudin, Phys. Rev. B 28, 1711 (1983).

${ }^{6}$ I. A. Viktorov, E. K. Grishchenko, and T. M. Kaekina, Akust. Zh. 9, 162 (1963) [Sov. Phys. Acoust. 9, 131 (1963)].

${ }^{7}$ Al. A. Kolomenskii and A. A. Maznev, Pis'ma Zh. Éksp. Teor. Fiz. 53, 403 (1991) [JETP Lett. 53, 423 (1991)]; Phys. Rev. B 48, 14502 (1993).

${ }^{8}$ R. E. Vines, S. Tamura, and J. P. Wolfe, Phys. Rev. Lett. 74, 2729 (1995); 75, 1873 (E) (1995).

${ }^{9}$ R. E. Vines, M. R. Hauser, and J. P. Wolfe, Z. Phys. B 98, 255 (1995).

${ }^{10}$ C. Höss and H. Kinder, Physica B 219\&220, 706 (1996).

${ }^{11}$ B. Taylor, H. J. Maris, Phys. Rev. Lett. 23, 416 (1969); H. J. Maris, J. Acoust. Soc. Am. 50, 812 (1971).

${ }^{12}$ G. A. Northrop and J. P. Wolfe, in Nonequilibrium Phonon Dy- namics, edited by W. E. Bron (Plenum, New York, 1985), Chap. 5.

${ }^{13}$ H. Shirasaki and T. Makimoto, J. Appl. Phys. 49, 658, 661 (1978); 50, 2795 (1979).

${ }^{14}$ S. Tamura and K. Honjo, Jpn. J. Appl. Phys. Suppl. (3), 20, 17 (1980); K. Honjo and S. Tamura (unpublished).

${ }^{15}$ R. E. Camley and A. A. Maradudin, Phys. Rev. B 27, 1959 (1983).

${ }^{16}$ S. Tamura and M. Yagi, Phys. Rev. B 49, 17378 (1994).

${ }^{17}$ T. C. Lim and G. W. Farnell, J. Acoust. Soc. Am. 45, 845 (1969).

${ }^{18} \mathrm{We}$ used the elastic constants $C_{11}=16.58, C_{12}=6.39$, and $C_{44}=7.93$ (in units of $10^{11} \mathrm{dyn} / \mathrm{cm}^{2}$ ) and mass density $\rho_{S}=2.33 \mathrm{~g} / \mathrm{cm}^{3}$ for silicon and mass density $\rho_{L}=0.786 \mathrm{~g} / \mathrm{cm}^{3}$ and longitudinal sound velocity $c_{L}=1168 \mathrm{~m} / \mathrm{s}$ for alcohol, and $\rho_{L}=1.0 \mathrm{~g} / \mathrm{cm}^{3}$ and $c_{L}=1483 \mathrm{~m} / \mathrm{s}$ for water.

${ }^{19}$ There is a solution for $c<c_{L}$ and the corresponding wave is localized to the interface on both the liquid and solid sides. For this solution [analogous to the Stoneley wave at solid-solid interface (see Ref. 3)] $\mathbf{k}_{\|}$is real, but we are not interested in this mode of waves.

${ }^{20}$ A. A. Maradudin, P. A. Flinn, and R. A. Coldwell-Horsfall, Ann. Phys. (N. Y.) 15, 360 (1961). 
${ }^{21}$ If we take a larger value for $z_{2}$, the region with finite $E_{L}$ for PSW becomes smaller on either side of the [110] direction.

${ }^{22}$ A. Ikehata, R. E. Vines, S. Tamura, and J. P. Wolfe, Physica B 219 \& 220, 710 (1996).

${ }^{23}$ M. R. Hauser, R. L. Weaver, and J. P. Wolfe, Phys Rev. Lett. 68, 2604 (1992); R. L. Weaver, M. R. Hauser, and J. P. Wolfe, Z. Phys. B 90, 27 (1993).
${ }^{24}$ In the [110] direction $\kappa^{(3)}$ is real, leading to the fact that all terms, except for the last one in Eq. (A16), are real and the last term is pure imaginary. But this time all terms corresponding to Eq. (A16) for the [100] propagation are pure imaginary and by dividing by $i$, the corresponding equation becomes real, which means that ${ }^{-} \Delta y_{1}^{\prime}$ for a finite $\delta$ is also real. 